\title{
Curvilinear Integral Theorem for $G$-Monogenic Mappings in the Algebra of Complex Quaternion
}

\author{
Tetyana KUZMENKO* \\ Institute of Mathematics of the National Academy of Science of Ukraine, \\ 3, Tereshchenkivs'ka st., Kyiv, Ukraine \\ kuzmenko.ts15@gmail.com
}

Keywords: quaternion algebra, $G$-monogenic mapping, curvilinear Cauchy integral theorem.

\begin{abstract}
For $G$-monogenic mappings taking values in the algebra of complex quaternion we prove a curvilinear analogue of the Cauchy integral theorem in the case where a curve of integration lies on the boundary of a domain of $G$-monogeneity.
\end{abstract}

\section{Introduction}

Let $\mathbb{H}(\mathbb{C})$ be the quaternion algebra over the field of complex numbers $\mathbb{C}$, whose basis consists of the unit 1 of the algebra and of the elements $I, J, K$ satisfying the multiplication rules:

$$
\begin{gathered}
I^{2}=J^{2}=K^{2}=-1, \\
I J=-J I=K, \quad J K=-K J=I, \quad K I=-I K=J .
\end{gathered}
$$

In the algebra $\mathbb{H}(\mathbb{C})$ there exists another basis $\left\{e_{1}, e_{2}, e_{3}, e_{4}\right\}$ such that multiplication table in a new basis can be represented as (see, e. g., [1])

\begin{tabular}{c||c|c|c|c|}
$\cdot$ & $e_{1}$ & $e_{2}$ & $e_{3}$ & $e_{4}$ \\
\hline \hline$e_{1}$ & $e_{1}$ & 0 & $e_{3}$ & 0 \\
\hline$e_{2}$ & 0 & $e_{2}$ & 0 & $e_{4}$ \\
\hline$e_{3}$ & 0 & $e_{3}$ & 0 & $e_{1}$ \\
\hline$e_{4}$ & $e_{4}$ & 0 & $e_{2}$ & 0 \\
\hline
\end{tabular}

The unit of the algebra can be decomposed as $1=e_{1}+e_{2}$.

Let us consider the vectors

$$
i_{1}=e_{1}+e_{2}, \quad i_{2}=a_{1} e_{1}+a_{2} e_{2}, \quad i_{3}=b_{1} e_{1}+b_{2} e_{2},
$$

$a_{k}, b_{k} \in \mathbb{C}, k=1,2$, which are linearly independent over the field of real numbers $\mathbb{R}$. It means that the equality $\alpha_{1} i_{1}+\alpha_{2} i_{2}+\alpha_{3} i_{3}=0$ for $\alpha_{1}, \alpha_{2}, \alpha_{3} \in \mathbb{R}$ holds if and only if $\alpha_{1}=\alpha_{2}=\alpha_{3}=0$.

In the algebra $\mathbb{H}(\mathbb{C})$ we consider the linear span $E_{3}:=\left\{\zeta=x i_{1}+y i_{2}+z i_{3}: x, y, z \in \mathbb{R}\right\}$ generated by the vectors $i_{1}, i_{2}, i_{3}$ over the field $\mathbb{R}$. A set $S \subset \mathbb{R}^{3}$ is associated with the set $S_{\zeta}:=\{\zeta=$ $\left.x i_{1}+y i_{2}+z i_{3}:(x, y, z) \in S\right\}$ in $E_{3}$. We also note that a topological property of a set $S_{\zeta}$ in $E_{3}$ understand as the same topological property of the set $S$ in $\mathbb{R}^{3}$. For example, we will say that a curve $\gamma_{\zeta} \subset E_{3}$ is homotopic to a point if $\gamma \subset \mathbb{R}^{3}$ is homotopic to a point, etc.

Let $\Omega$ be a domain in $\mathbb{R}^{3}$.

We say (see [2]) that a continuous mapping $\Phi: \Omega_{\zeta} \rightarrow \mathbb{H}(\mathbb{C})\left(\right.$ or $\left.\widehat{\Phi}: \Omega_{\zeta} \rightarrow \mathbb{H}(\mathbb{C})\right)$ is right-Gmonogenic (or left-G-monogenic) in a domain $\Omega_{\zeta} \subset E_{3}$, if $\Phi$ (or $\widehat{\Phi}$ ) is differentiable in the sense of the Gâteaux at every point of $\Omega_{\zeta}$, i. e. for every $\zeta \in \Omega_{\zeta}$ there exists an element $\Phi^{\prime}(\zeta) \in \mathbb{H}(\mathbb{C})$ (or $\left.\widehat{\Phi}^{\prime}(\zeta) \in \mathbb{H}(\mathbb{C})\right)$ such that

$$
\lim _{\varepsilon \rightarrow 0+0}(\Phi(\zeta+\varepsilon h)-\Phi(\zeta)) \varepsilon^{-1}=h \Phi^{\prime}(\zeta) \quad \forall h \in E_{3}
$$




$$
\text { (or } \left.\lim _{\varepsilon \rightarrow 0+0}(\widehat{\Phi}(\zeta+\varepsilon h)-\widehat{\Phi}(\zeta)) \varepsilon^{-1}=\widehat{\Phi}^{\prime}(\zeta) h \quad \forall h \in E_{3}\right) .
$$

The Cauchy integral theorems for holomorphic functions of the complex variable are fundamental results of the classical complex analysis. Analogues of these results are also important tools in the quaternionic analysis.

In the paper [3] were established some analogues of classical integral theorems of the theory of analytic functions of the complex variable: the surface and curvilinear Cauchy integral theorems and the Cauchy integral formula. The Morera theorem was proved in the paper [4]. Taylor's and Laurent's expansions of $G$-monogenic mappings are obtained in [5].

Namely, in the paper [3] was proved a curvilinear analogue of the Cauchy integral theorem in the case where a curve of integration lies in a domain of $G$-monogeneity.

In the present paper we prove a curvilinear Cauchy integral theorem for $G$-monogenic mappings in the case where a curve of integration lies on the boundary of a domain of $G$-monogeneity.

\section{The main result}

Let $\gamma$ be a Jordan rectifiable curve in $\mathbb{R}^{3}$. For a continuous mapping $\Psi: \gamma_{\zeta} \rightarrow \mathbb{H}(\mathbb{C})$ of the form

$$
\Psi(\zeta)=\sum_{k=1}^{4}\left(U_{k}(x, y, z)+i V_{k}(x, y, z)\right) e_{k},
$$

where $(x, y, z) \in \gamma$ and $U_{k}: \gamma \rightarrow \mathbb{R}, V_{k}: \gamma \rightarrow \mathbb{R}$, we define integrals along a Jordan rectifiable curve $\gamma_{\zeta}$ by the equalities

$$
\begin{aligned}
\int_{\gamma_{\zeta}} d \zeta & \Psi(\zeta):=\sum_{k=1}^{4} e_{k} \int_{\gamma} U_{k}(x, y, z) d x+\sum_{k=1}^{4} i_{2} e_{k} \int_{\gamma} U_{k}(x, y, z) d y+ \\
& +\sum_{k=1}^{4} i_{3} e_{k} \int_{\gamma} U_{k}(x, y, z) d z+i \sum_{k=1}^{4} e_{k} \int_{\gamma} V_{k}(x, y, z) d x+ \\
& +i \sum_{k=1}^{4} i_{2} e_{k} \int_{\gamma} V_{k}(x, y, z) d y+i \sum_{k=1}^{4} i_{3} e_{k} \int_{\gamma} V_{k}(x, y, z) d z
\end{aligned}
$$

and

$$
\begin{aligned}
& \int_{\gamma_{\zeta}} \Psi(\zeta) d \zeta:=\sum_{k=1}^{4} e_{k} \int_{\gamma} U_{k}(x, y, z) d x+\sum_{k=1}^{4} e_{k} i_{2} \int_{\gamma} U_{k}(x, y, z) d y+ \\
& \quad+\sum_{k=1}^{4} e_{k} i_{3} \int_{\gamma} U_{k}(x, y, z) d z+i \sum_{k=1}^{4} e_{k} \int_{\gamma} V_{k}(x, y, z) d x+ \\
& +i \sum_{k=1}^{4} e_{k} i_{2} \int_{\gamma} V_{k}(x, y, z) d y+i \sum_{k=1}^{4} e_{k} i_{3} \int_{\gamma} V_{k}(x, y, z) d z,
\end{aligned}
$$

where $d \zeta:=i_{1} d x+i_{2} d y+i_{3} d z$.

In the paper [3] for right- $G$-monogenic mappings was obtained the following analogue of the Cauchy integral theorem. 
Theorem 1 [3]. Let $\Phi: \Omega_{\zeta} \rightarrow \mathbb{H}(\mathbb{C})$ be a right-G-monogenic mapping in a domain $\Omega_{\zeta}$. Then for every closed Jordan rectifiable curve $\gamma_{\zeta}$ homotopic to a point in $\Omega_{\zeta}$, the following equality is true:

$$
\int_{\gamma_{\zeta}} d \zeta \Phi(\zeta)=0
$$

Below we establish sufficient conditions for the curve $\gamma_{\zeta}$ lying on the boundary $\partial \Omega_{\zeta}$ of a domain $\Omega_{\zeta}$ such that the equality (3) holds. For this goal we apply a scheme of the proof of Theorem 4 of [6] for $G$-monogenic mappings.

Let on a boundary $\partial \Omega_{\zeta}$ of the domain $\Omega_{\zeta}$ given closed Jordan rectifiable curve $\gamma_{\zeta} \equiv \gamma_{\zeta}(t)$, where $0 \leq t \leq 1$, homotopic to an interior point $\zeta_{0} \in \Omega_{\zeta}$. It means that there exists the mapping $H(s, t)$ continuous on the square $[0,1] \times[0,1]$, such that $H(0, t)=\gamma_{\zeta}(t), H(1, t) \equiv \zeta_{0}$, and all curves $\gamma_{\zeta}^{s} \equiv \gamma_{\zeta}^{s}(t):=\{\zeta=H(s, t): 0 \leq t \leq 1\}$ for $0<s<1$ are contained in the domain $\Omega_{\zeta}$.

Consider also the curves $\Gamma_{\zeta}^{t} \equiv \Gamma_{\zeta}^{t}(s):=\{\zeta=H(s, t): 0 \leq s \leq 1\}$. Denote by $\Gamma\left[\zeta_{1}, \zeta_{2}\right]$ arc of Jordan oriented rectifiable curve, beginning at the point $\zeta_{1}$ and ending at the point $\zeta_{2}$, and denote by the mes a linear Lebesgue measure of a rectifiable curve.

Let us consider the algebra $\widetilde{\mathbb{H}}(\mathbb{R})$ with the basis $\left\{e_{k}, i e_{k}\right\}_{k=1}^{4}$ over the field $\mathbb{R}$ which is isomorphic to the algebra $\mathbb{H}(\mathbb{C})$ over the field $\mathbb{C}$. In the algebra $\widetilde{\mathbb{H}}(\mathbb{R})$ there exist another basis $\left\{i_{k}\right\}_{k=1}^{8}$, where the vectors $i_{1}, i_{2}, i_{3}$ are the same as in the equalities (1).

For the element $a:=\sum_{k=1}^{8} a_{k} i_{k}, a_{k} \in \mathbb{R}$, we define the Euclidian norm

$$
\|a\|:=\sqrt{\sum_{k=1}^{8} a_{k}^{2}} .
$$

Accordingly, $\|\zeta\|=\sqrt{x^{2}+y^{2}+z^{2}}$ and $\left\|i_{1}\right\|=\left\|i_{2}\right\|=\left\|i_{3}\right\|=1$.

Using Theorem of equivalents of norms, for the element $b:=\sum_{k=1}^{4}\left(b_{1 k}+i b_{2 k}\right) e_{k}, b_{1 k}, b_{2 k} \in \mathbb{R}$, we have the following inequalities:

$$
\left|b_{1 k}+i b_{2 k}\right| \leq \sqrt{\sum_{k=1}^{4}\left(b_{1 k}^{2}+b_{2 k}^{2}\right)} \leq c\|b\|,
$$

where $c$ is a positive constant does not dependent on $b$.

Theorem 2. Suppose that $\Phi: \bar{\Omega}_{\zeta} \rightarrow \mathbb{H}(\mathbb{C})$ is a continuous mapping in the closure $\bar{\Omega}_{\zeta}$ of a domain $\Omega_{\zeta}$ and $\Phi$ is right-G-monogenic in $\Omega_{\zeta}$. Suppose also that $\gamma_{\zeta} \subset \partial \Omega_{\zeta}$ is any closed Jordan rectifiable curve homotopic to a point $\zeta_{0} \in \Omega_{\zeta}$ such that the curves of the family $\left\{\Gamma_{\zeta}^{t}: 0 \leq t \leq 1\right\}$ are rectifiable and the set $\left\{\operatorname{mes} \gamma_{\zeta}^{s}: 0 \leq s \leq 1\right\}$ is bounded. Then the equality (3) is true.

Proof. Let $\varepsilon>0$. We fix the number $\rho \in\left(0, \frac{1}{2}\right.$ mes $\left.\gamma_{\zeta}\right)$ such that for arbitrary $\zeta_{1}, \zeta_{2} \in \bar{\Omega}_{\zeta}$ from the condition $\left\|\zeta_{1}-\zeta_{2}\right\|<2 \rho$ follows the inequality

$$
\left\|\Phi\left(\zeta_{1}\right)-\Phi\left(\zeta_{2}\right)\right\|<\varepsilon
$$

Since the mapping $H$ is uniformly continuous on the square $[0,1] \times[0,1]$, then there exists $\delta>0$ such that for all $s \in(0, \delta)$ and $t, t^{\prime} \in[0,1]:\left|t-t^{\prime}\right|<\delta$ the inequality $\left|H(0, t)-H\left(s, t^{\prime}\right)\right|<\rho$ is true.

Let numbers $0=t_{0}<t_{1}<\ldots<t_{n}<1$ such that for corresponding points $\zeta_{0, k}:=H\left(0, t_{k}\right)$ of the curve $\gamma_{\zeta}$ the following relations are fulfilled

$$
\operatorname{mes} \gamma_{\zeta}\left[\zeta_{0, k}, \zeta_{0, k+1}\right]=\rho \quad \text { for } \quad k=\overline{0, n-1},
$$




$$
\operatorname{mes} \gamma_{\zeta}\left[\zeta_{0, n}, \zeta_{0,0}\right] \leq \rho .
$$

It is obvious that $2 \leq n \leq\left[\frac{\operatorname{mes} \gamma_{\zeta}}{\rho}\right]+1$.

Let us consider the points $\zeta_{s, k}:=H\left(s, t_{k}\right)$ of the curve $\gamma_{\zeta}^{s}$ and the curves

$$
\Upsilon_{[k]}^{s}:=\gamma_{\zeta}\left[\zeta_{0, k}, \zeta_{0, k+1}\right] \cup \Gamma_{\zeta}^{t_{k+1}}\left[\zeta_{0, k+1}, \zeta_{s, k+1}\right] \cup \gamma_{\zeta}^{s}\left[\zeta_{s, k+1}, \zeta_{s, k}\right] \cup \Gamma_{\zeta}^{t_{k}}\left[\zeta_{s, k}, \zeta_{0, k}\right]
$$

for $k=\overline{0, n}$, where $\zeta_{s, n+1}:=\zeta_{s, 0}$ for $0 \leq s \leq 1$, setting that the orientation of curves $\Upsilon_{[k]}^{s}$ is induced by orientation of the curve $\gamma_{\zeta}$.

Let $s \in(0, \delta)$. Since for all $\zeta \in \Upsilon_{[k]}^{s}$ the inequality $\left\|\zeta-\zeta_{0, k}\right\| \leq 2 \rho$ is true, then by Theorem 2 [3], Lemma 4.1 [4] and the inequality (4), we have

$$
\begin{gathered}
\left\|\int_{\gamma_{\zeta}} d \zeta \Phi(\zeta)\right\|=\left\|\sum_{k=0}^{n} \int_{\Upsilon_{[k]}^{s}} d \zeta\left(\Phi(\zeta)-\Phi\left(\zeta_{0, k}\right)\right)\right\| \leq \\
\leq c \sum_{k=0}^{n} \int_{\Upsilon_{[k]}^{s}}\|d \zeta\|\left\|\Phi(\zeta)-\Phi\left(\zeta_{0, k}\right)\right\| \leq c \varepsilon \sum_{k=0}^{n} \operatorname{mes} \Upsilon_{[k]}^{s} \leq \\
\leq c \varepsilon\left(\operatorname{mes} \gamma_{\zeta}+\operatorname{mes} \gamma_{\zeta}^{s}+2(n+1) \max _{k=0, n} \operatorname{mes} \Gamma_{\zeta}^{t_{k}}\left[\zeta_{s, k}, \zeta_{0, k}\right]\right) \leq \\
\leq M \varepsilon\left(1+\frac{1}{\rho} \underset{k=\overline{0, n}}{\max } \operatorname{mes} \Gamma_{\zeta}^{t_{k}}\left[\zeta_{s, k}, \zeta_{0, k}\right]\right)
\end{gathered}
$$

and a constant $M$ does not depend on $\varepsilon$ and $\rho$.

Passing to the limit in the inequality (5) as $s \rightarrow 0$, we have the inequality

$$
\left\|\int_{\gamma_{\zeta}} d \zeta \Phi(\zeta)\right\| \leq M \varepsilon .
$$

Now passing to the limit in the last inequality as $\varepsilon \rightarrow 0$, we obtain the equality (3). The Theorem is proved.

The similar statement is true for the left- $G$-monogenic mappings.

Theorem 3. Suppose that $\widehat{\Phi}: \bar{\Omega}_{\zeta} \rightarrow \mathbb{H}(\mathbb{C})$ is a continuous mapping in the closure $\bar{\Omega}_{\zeta}$ of a domain $\Omega_{\zeta}$ and $\widehat{\Phi}$ is left-G-monogenic in $\Omega_{\zeta}$. Suppose also that $\gamma_{\zeta} \subset \partial \Omega_{\zeta}$ is any closed Jordan rectifiable curve homotopic to a point $\zeta_{0} \in \Omega_{\zeta}$ such that the curves of the family $\left\{\Gamma_{\zeta}^{t}: 0 \leq t \leq 1\right\}$ are rectifiable and the set $\left\{\right.$ mes $\left.\gamma_{\zeta}^{s}: 0 \leq s \leq 1\right\}$ is bounded. Then the following equality is true:

$$
\int_{\gamma_{\zeta}} \widehat{\Phi}(\zeta) d \zeta=0 .
$$

* This work was supported by the Ministry of Education and Science of Ukraine (project "Monogenic functions in Banach algebras and boundary value problems of analysis and mathematical physics 


\section{References}

[1] E. Cartan, Les groupes bilinéares et les systèmes de nombres complexes, Annales de la faculté des sciences de Toulouse. 12:1 (1898) 1-64.

[2] V. S. Shpakivskyi, T. S. Kuzmenko, On one class of quaternionic mappings. Ukr. Math. J. 68:1 (2016) 117-130.

[3] V. S. Shpakivskyi, T. S. Kuzmenko, Integral theorems for the quaternionic $G$-monogenic mappings. An. Şt. Univ. Ovidius Constanţa. 24:2 (2016).

[4] V. S. Shpakivskyi, T. S. Kuzmenko, On monogenic mappings of the quaternionic variable. Ukr. Math. Bull. (submitted).

[5] T. S. Kuzmenko, Power and Laurent series in the algebra of complex quaternion. Zb. Pr. Inst. Mat. NAN Ukr. 12:3 (2015) 164-174 (Ukrainian).

[6] S. A. Plaksa, V.S. Shpakivskyi, Integral theorems for differentiable functions in a threedimensional harmonic algebra. Reports of the NAS of Ukraine. 5 (2010) 23-30 (Russian). 